\title{
Metal ion influence on eumelanin fluorescence and structure
}

\author{
Jens-Uwe Sutter and David J S Birch* \\ Photophysics Group, Department of Physics, SUPA, \\ University of Strathclyde, Glasgow, G4 0NG, Scotland \\ *E-mail: djs.birch@strath.ac.uk
}

\begin{abstract}
Melanin has long been thought to have an unworkably weak and complex fluorescence, but here we study its intrinsic fluorescence in order to demonstrate how metal ions can be used to control the rate of formation, constituents and structure of eumelanin formed from the well-known laboratory auto-oxidation of 3, 4-dihydroxy-L-phenylalanine (LDOPA). The effect on eumelanin absorption and fluorescence of a range of solvated metal ions is reported including $\mathrm{Cu}$, $\mathrm{Zn}, \mathrm{Ni}, \mathrm{Na}$ and $\mathrm{K}$. Monovalent cations and $\mathrm{Zn}$ have little effect, but the effect of transition metal cations can be considerable. For example, at $\mathrm{pH} 10$, copper ions are shown to accelerate the onset of eumelanin formation, but not the rate of formation once it commences, and simplify the usual complex structure and intrinsic fluorescence of eumelanin in a way that is consistent with an increased abundance of 5,5-dihydroxyindole-2-carboxylic acid (DHICA). The presence of a dominant $6 \mathrm{~ns}$ fluorescence decay time at $480 \mathrm{~nm}$, when excited at $450 \mathrm{~nm}$ describes a distinct photophysically species, which we tentatively assign to small oligomers. Copper is well-known to normally quench fluorescence, but increasing amounts of copper surprisingly leads to an increase in the fluorescence decay time of eumelanin, while reducing the fluorescence intensity, suggesting copper modification of the excited state. Such results have bearing on diverse areas. The most accepted morphology for melanin is that of a graphite-like sheet structure, and one which readily binds metal ions, an interaction that is thought to have an important, though as yet unclear bearing on several areas of medicine including neurology. There is also increasing interest in bio-mimicry by preparing and labelling sheet structures with metal ions for new electronic and photonic materials.
\end{abstract}

Keywords: Melanin, eumelanin, 3, 4-dihydroxy-L-phenylalanine, L-DOPA, copper ions, fluorescence, polymerization

\section{Introduction}

Metal ions play many important roles in human metabolism, including acting as reaction centres in protein and enzymes, and facilitating catalysis, transport, signalling and aggregation. In addition to being vital for normal biological function, metal ions (and transition metal ions in particular), in the absence of regulation, are thought to be implicated in the generation of reactive species such as free radicals, that can lead to oxidative stress and the cell damage associated with several diseases. These include neurological conditions such as Alzheimer's, Parkinson's and Huntington's. In addition, transition metal ions are known to accelerate the aggregation of certain biological systems. For example, the aggregation of beta-amyloid, that is associated with the origins of Alzheimer's disease, is accelerated by copper and zinc ions, which are also found on autopsy at abnormally high concentrations $(\sim \mathrm{mM})$ in neuro senile plaques and fibrils [1]. Therapies capable of inhibiting and reversing this aggregation are the subject of global research [2].

Here we focus on melanin, a ubiquitous pigment perhaps best known for its photo-protection of skin, but also implicated in melanoma, the most aggressive form of skin cancer. The synthesis of the most common natural form of melanin (eumelanin) occurs in melanosomes, which are organelles within melanocytes, and proceeds from the amino acid Ltyrosine though a series of chemical and enzymatic steps facilitated by the copper-containing glycoprotein tyrosinase [3]. A very close representation of natural eumelanin is readily synthesized in the laboratory from the auto-oxidation of 3, 4dihydroxy-L-phenylalanine (L-DOPA) [3]. Both natural and synthetic melanin are understood to be heteropolymers composed predominantly of 5,6- dihydroxyindole (DHI) and 5,5-dihydroxyindole-2-carboxylic acid (DHICA) units and their redox forms. The evidence points towards these constituents being arranged in a $\pi$-stacked graphite-like sheet structure [4-7], although the actual structural arrangement of the constituents remains unknown [8]. Figure 1 summarizes the main stages of the synthesis [3] we will consider. 
There is an interesting range of disease correlations around melanin, including those in neurology and cancer. For example Parkinson's disease is associated with abnormally low levels of neuromelanin, increased risk of melanoma [9], and can be treated by L-DOPA, which has been shown to disaggregate amyloid fibrils $\alpha$-synuclein and beta-amyloid that are known to be associated with Parkinson's disease and Alzheimer's disease respectively [10]. Also, analogous to betaamyloid, organic accelerators and inhibitors of eumelanin aggregation are being investigated [11].

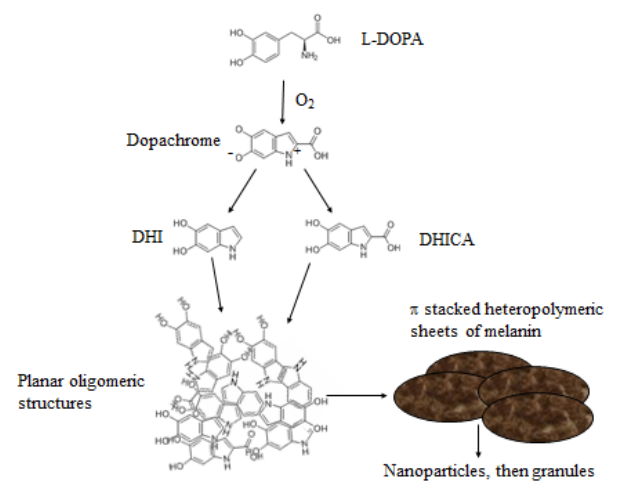

Figure 1. Simplified auto-oxidation scheme of L-DOPA to produce eumelanin

One difference between natural and synthetic melanin is the presence of copper in the former. Correlating with this is the greater relative abundance of DHICA with respect to DHI in natural melanin (50\%) as compared to melanin synthesized from L-DOPA (10\%), and in eumelanin synthesized at higher than physiological $\mathrm{pH}$ [12]. Moreover, addition of metal ions, particularly $\mathrm{Cu}^{2+}$, has previously been found to accelerate the formation of eumelanin from L-DOPA. Cu ${ }^{2+}$ has been suggested to bind predominantly to hydroxyl groups at $\mathrm{pH} 7-11[13]$ and increase the concentration of DHICA in synthetic eumelanin formed from L-DOPA in the presence of tyrosinase by catalyzing the re-arrangement of the intermediate dopachrome in favour of DHICA rather than DHI [14].

Recently melanin has taken on a wider research interest beyond biology in terms of a possible technology platform for memory, switching and photonics $[15,16]$. Such potential opportunities have been realized largely because of melanin's unique combination of physical properties, which include a broad spectral absorption, low fluorescence quantum yield, paramagnetism, semiconducting and hydration-dependent conduction and photoconduction [8]. Although melanin structural modification for applications in technology can in principle be achieved using $\mathrm{pH}$ and dopants such as metal ions, melanin synthesis is complex and the pathway to the end products poorly charted.

Indeed, while eumelanin is well-known to strongly bind metal ions the effect on the formation kinetics, structure and associated fluorescence have been little explored. In this paper we investigate such effects in respect of findings that have bearing on biology as well as the modification of eumelanin's sheet structures for potential use in bio-mimicked technology.

Although ideal for tracking changes in many biological processes, fluorescence has not been the technique of choice in melanin research and application because of melanin's low fluorescence quantum yield, overlapping spectral components and complex fluorescence decay kinetics. Nevertheless here we report the usefulness of fluorescence spectroscopy in monitoring and characterizing the effect of metal ions in modifying eumelanin's structure and report how copper ions in particular are effective in simplifying the end products and concomitant fluorescence photophysics.

\section{Experimental}

While eumelanin is formed from L-DOPA over a wide range of $\mathrm{pH}$ through auto-oxidation, the time it takes for the reaction to conclude is generally shortened at alkaline $\mathrm{pH}$ with respect to physiological $\mathrm{pH}$. A stock solution of L-DOPA 
at $1 \mathrm{mM}$ concentration was first mixed by ultra-sonication for $30 \mathrm{~min}$ and measurements were typically taken at an LDOPA concentration of $40 \mu \mathrm{M}$. Metal ion salts were added from aqueous stock solution to a final concentration range of $4 \mu \mathrm{M}$ to $4 \mathrm{mM}$. Salts used were $\mathrm{CuSO}_{4}, \mathrm{CuCl}_{2}, \mathrm{ZnCl}_{2}, \mathrm{NiCl}_{2}, \mathrm{NaCl}$ and $\mathrm{KCl}$. DHICA was purchased from Toronto Research Chemicals Inc, Canada. All other chemicals were obtained at the highest available purity from Sigma Aldrich, UK. To measure fluorescence spectra we used a Horiba Scientific FluoroLog fluorimeter. Absorption measurements were performed using a Jasco V-660 Spectrophotometer. To obtain kinetics of fluorescence and absorption, spectral measurements were at a few minute intervals until the spectrum reached steady-state. To initiate the start of the eumelanin synthesis $50 \mu \mathrm{l}$ ammoniac was added to adjust to $\mathrm{pH} 10$. Fluorescence decay measurements were performed using time-correlated single-photon counting [17] with a Horiba Jobin Yvon IBH FluoroCube. Excitation was with either a $1 \mathrm{MHz}$ NanoLED [18] laser diode emitting at $452 \mathrm{~nm}$ and < $200 \mathrm{ps}$ fwhm or a $40 \mathrm{MHz}$ DeltaDiode at $378 \mathrm{~nm}$.

\section{Results and discussion}

\subsection{Absorption spectra}

Given the weakness of melanin fluorescence, absorption studies were the basis of much of the early structural information that could be gleaned. Melanin is characterized by its broad-band absorption spectrum covering the visible spectrum all through to the near UV. The temporal evolution of this absorption spectrum during the formation of eumelanin from L-DOPA reveals a highly organized process with characteristic phases. Within the first minute of adjusting the $\mathrm{pH}$ to 10 two absorption peaks appear at $240 \mathrm{~nm}$ and $295 \mathrm{~nm}$ that are typical of indole structures (see figure 2(a)). During the first phase these peaks rise with the peak at $295 \mathrm{~nm}$ displaying a slight bathochromic shift. At, for examples, $40 \mu \mathrm{M}$ L-DOPA and $\mathrm{pH} 10$ the first phase lasts for about $12 \mathrm{~min}$. In a second phase isobestic points form within the absorption spectrum and the absorption evolves towards the characteristic melanin broad-band absorption spectrum (figure 2(a)) with a new peak initially appearing that centres at $\sim 440 \mathrm{~nm}$. The identification of these peaks is today still not totally unambiguous. For example, $440 \mathrm{~nm}$ is close to, but not identical to the $475 \mathrm{~nm}$ absorption reported for dopachrome at pH $5.5[19,20]$. Such a long wavelength of $440 \mathrm{~nm}$ would not seem to be attributable to monomeric indole structures such as DHI and DHICA, but might represent their extended polymeric $\pi$ conjugations. The absorption peak $~ 290 \mathrm{~nm}$, which undergoes with time a bathochromic shift in figure 2(a), has also been attributed to both DHICA $[19,20]$ and dopachrome [21]. However, the latter is an unstable intermediate and it is generally agreed that DHI, DHICA and their redox forms are the dominant stable species [8]. The absorption peak measured for DHICA alone at $310 \mathrm{~nm}$ in Figure 2(b) confirms the "end-point" species absorbing in figure 2(a) to be DHICA.

This highly organized process in eumelanin formation is greatly altered in the presence of metal ions. For example, with the addition of $400 \mu \mathrm{M} \mathrm{Cu}^{2+}$ the initial phase showing the formation of DHICA from dopachrome is either absent or too rapid to be observed and the absorption is strongly shifted towards shorter wavelengths with the peak at $440 \mathrm{~nm}$ already present at the outset (figure 2(c)). In addition the final absorption spectrum is more akin to the structureless and

(a)

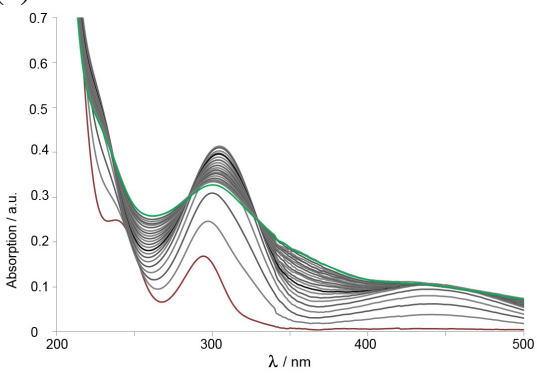

(b)

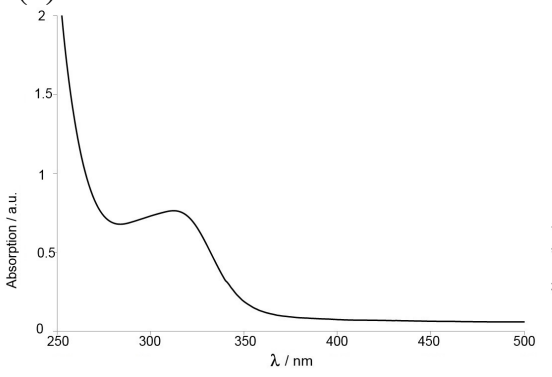

(c)

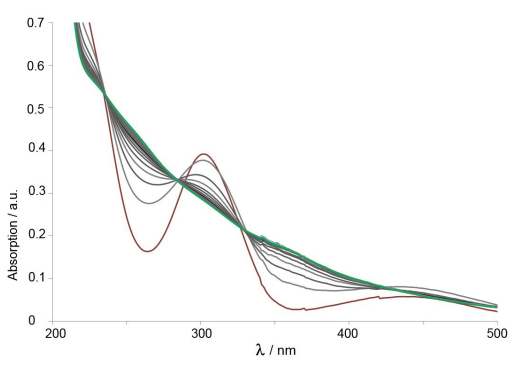

Figure 2. Evolution of absorption spectra at pH 10 for eumelanin synthesis. (a) For $40 \mu \mathrm{M}$ L-DOPA, (b) DHICA absorption spectrum, (c) melanin formed from $40 \mu \mathrm{M} \mathrm{L-DOPA}$ in the presence of $400 \mu \mathrm{M} \mathrm{CuSO}_{4}$. For figures (a) and (c) the first measurement marked in red is at 1 min after adjusting the $\mathrm{pH}$ to 10, the measurement after 30 min is marked in black, and the final spectrum recorded after $90 \mathrm{~min}$ is marked in green. Measurements in between these times are at 4 min interval and are in grey. For 2(a) the peak at $\sim 305 \mathrm{~nm}$ is reached after $22 \mathrm{~min}$. 
exponential-like continuum of natural eumelanin with increasing absorption at shorter wavelengths. Figure 3 compares the effect of $\mathrm{Cu}^{2+}, \mathrm{Ni}^{2+}, \mathrm{Zn}^{2+}$ and $\mathrm{K}^{+}$on the eumelanin synthesis in comparison to having no metal ions present. $\mathrm{Cu}^{2+}$ leads to an increase in absorption apart from above $375 \mathrm{~nm}$ suggesting arrestment of the eumelanin synthesis before extended $\pi$ conjugations of polymeric forms of indoles are formed. In the case of $400 \mu \mathrm{M} \mathrm{Cu}^{2+}$ the absorption at $480 \mathrm{~nm}$ is decreased by $70 \%$. With $400 \mu \mathrm{M} \mathrm{Ni}^{2+}$ the reduction is even more pronounced at $80 \%$. Other physiologically relevant ions like $\mathrm{Zn}^{2+}$ or $\mathrm{K}^{+}$do very little to alter the absorption of eumelanin. Indeed, there is no marked decrease in eumelanin absorption at wavelengths $>375 \mathrm{~nm}$, suggesting less interaction. For example $400 \mu \mathrm{M} \mathrm{Zn}^{2+}$ only decreases eumelanin absorption at $480 \mathrm{~nm}$ by $6 \%$ and $400 \mu \mathrm{M} \mathrm{K}^{+}$leads to no reduction. Only $\mathrm{Cu}^{2+}$ causes a dramatic change in the final eumelanin absorption spectrum at wavelengths $<350 \mathrm{~nm}$. Absorption at $275 \mathrm{~nm}$ is increased by $250 \%$ in the presence of $\mathrm{Cu}^{2+}$. The presence of $\mathrm{Ni}^{2+}$ leads to a slight decrease in absorption over a broad range from $230 \mathrm{~nm}$ to $500 \mathrm{~nm}$.

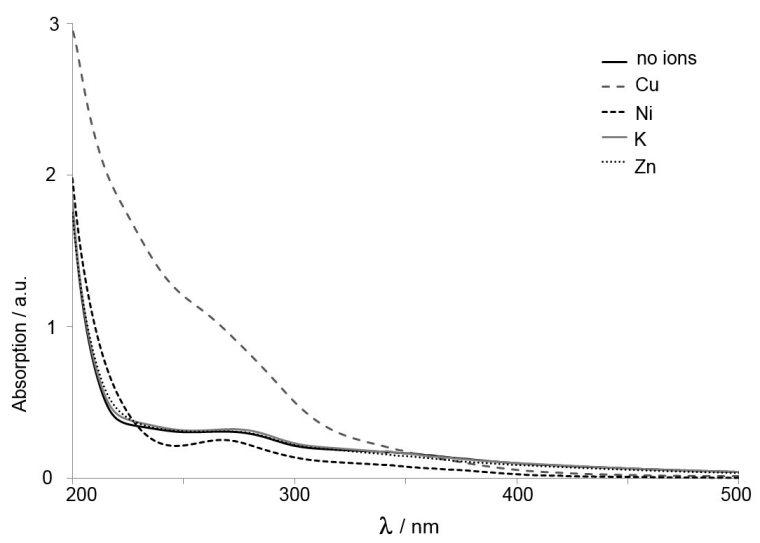

Figure 3. The absorption of melanin formed in the absence or presence of different metal ions. Metals have been added at the start of the melanin formation as solutions of $\mathrm{CuSO}_{4}, \mathrm{NiCl}_{2}, \mathrm{ZnCl}_{2}$ and $\mathrm{KCl}$ each at a concentration of $400 \mu \mathrm{M}$. The melanin formation took place at $\mathrm{pH} 10$ and the absorption spectra were measured 4 days after the onset of the reaction.

\subsection{Fluorescence spectra}

The changes in the absorption spectrum of eumelanin induced by metal ions are reflected in the fluorescence spectrum. Exciting at $450 \mathrm{~nm}$ the relative fluorescence intensity in the presence of $\mathrm{Cu}^{2+}$ and $\mathrm{Ni}^{2+}$ ions mirrors the arrestment of species responsible for the absorptions above $375 \mathrm{~nm}$ (figure 4).

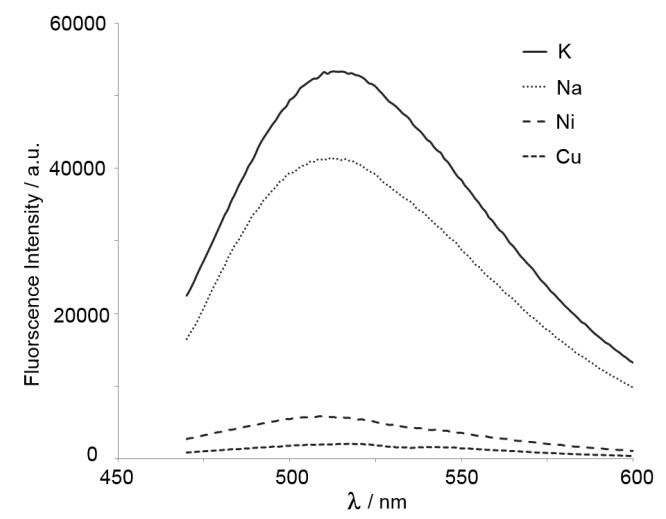

Figure 4. The fluorescence emission of eumelanin in the presence of ions excited at $450 \mathrm{~nm}$. Metal ions have been added as solutions of $\mathrm{CuSO}_{4}, \mathrm{NiCl}_{2}, \mathrm{NaCl}$ and $\mathrm{KCl}$, each at a concentration of $400 \mu \mathrm{M}$. The eumelanin formation took place at $\mathrm{pH} 10$ and the spectra were measured 4 days after the onset of the reaction. 
In the absence of copper the formation of melanin yields a characteristic peak around $520 \mathrm{~nm}$ (figure 5(a)) that has previously been loosely attributed to polymer structures [22]. Adding $\mathrm{Cu}^{2+}$ to the reaction changes the evolution of the spectra [23]. At a $4 \mu \mathrm{M} \mathrm{CuSO}_{4}$ concentration we observe fluorescence rapidly rising at $480 \mathrm{~nm}$ (Fig 5(b)). This becomes more pronounced with increasing $\mathrm{Cu}^{2+}$ concentration. At $4 \mathrm{mM} \mathrm{CuSO}_{4}$ the initial peak at $530 \mathrm{~nm}$ nearly vanishes and the emission at $480 \mathrm{~nm}$ becomes dominant (Fig 5(c)) demonstrating how copper ions significantly modify the synthesis.

(a)

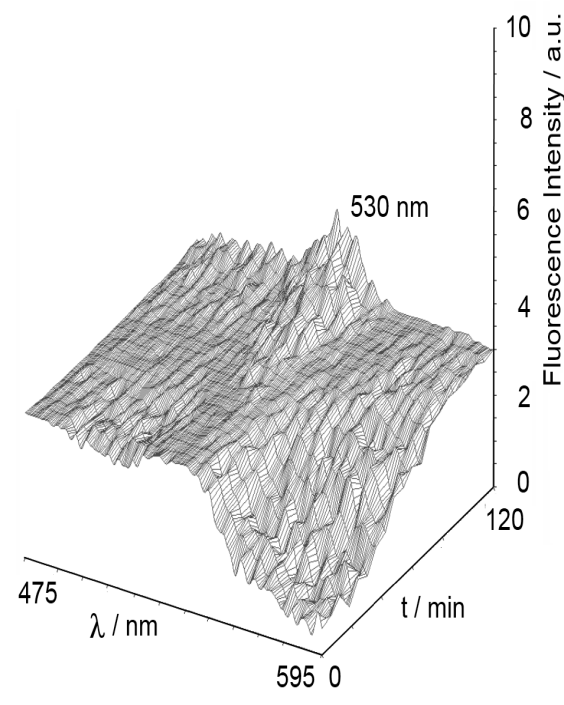

(b)

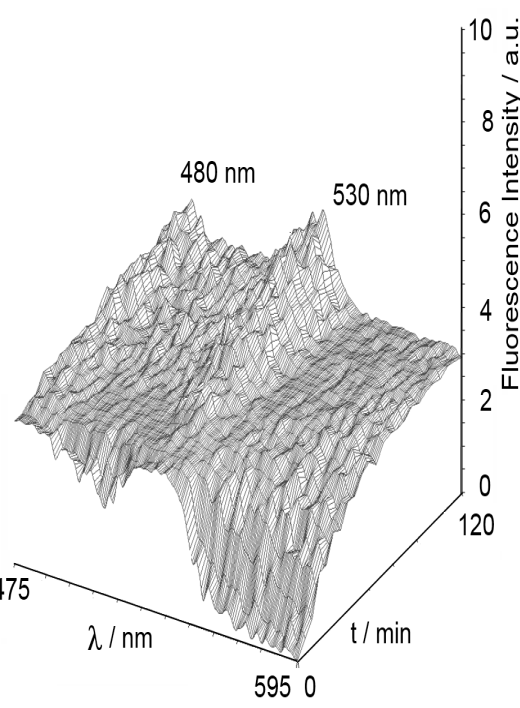

(c)

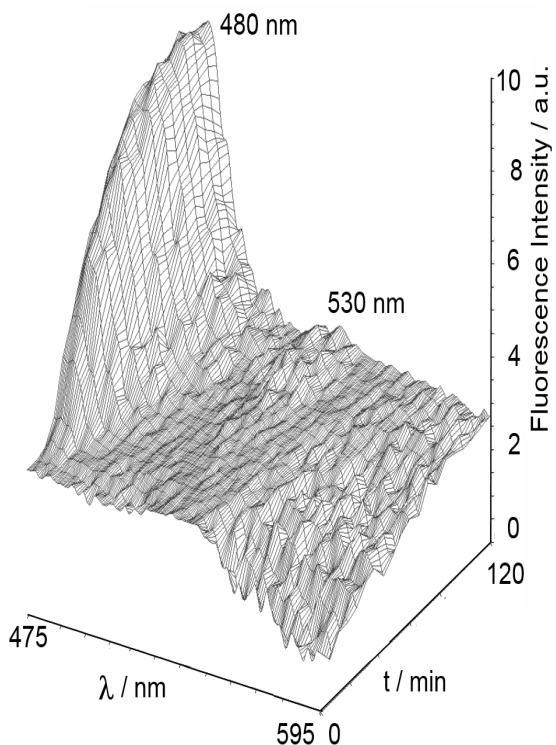

Figure 5. The evolution of eumelanin intrinsic fluorescence excited at $450 \mathrm{~nm}$. (a) No copper present (b) $4 \mu \mathrm{M}$. CuSO (c) $4 \mathrm{mM} . \mathrm{CuSO}_{4}[23]$.

Exciting the eumelanin at $375 \mathrm{~nm}$ as it forms reveals a more complex emission spectrum than at $450 \mathrm{~nm}$. Monitoring fluorescence from a solution of $40 \mu \mathrm{M}$ L-DOPA over 1 hour after shifting to $\mathrm{pH} 10$ shows an initial fluorescence peak around $530 \mathrm{~nm}$ and after about 10 min a distinct peak at $460 \mathrm{~nm}$ develops and the initial peak at $530 \mathrm{~nm}$ shifts towards shorter wavelength (figure 6(a)), eventually being less intense than the peak at $460 \mathrm{~nm}$. The presence of $400 \mu \mathrm{M} \mathrm{Cu} \mathrm{u}^{2+}$ leads to the omission of the initial peak at 530nm (figure 6(b)) and this is consistent with when exciting at $450 \mathrm{~nm}$ (figure (5)). Over the course of 1 hour fluorescence slowly rises but remains about $85 \%$ below the fluorescence without $\mathrm{Cu}^{2+}$. Such effects are less pronounced with a lower concentration of $40 \mu \mathrm{M} \mathrm{Cu}^{2+}$. The presence of $\mathrm{Cu}^{2+}$ leads to the formation of melanin with much increased absorption and decreased fluorescence characteristics. Both $\mathrm{K}^{+}$as well as $\mathrm{Zn}^{2+}$ had little influence on melanin fluorescence.

(a)

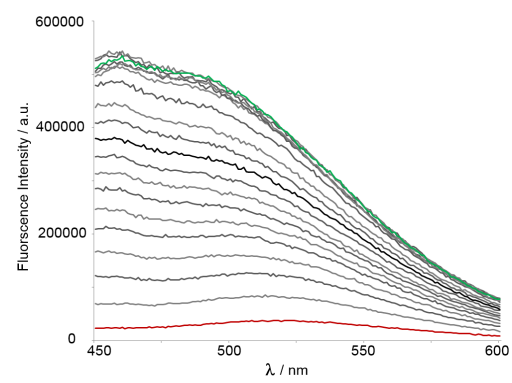

(b)

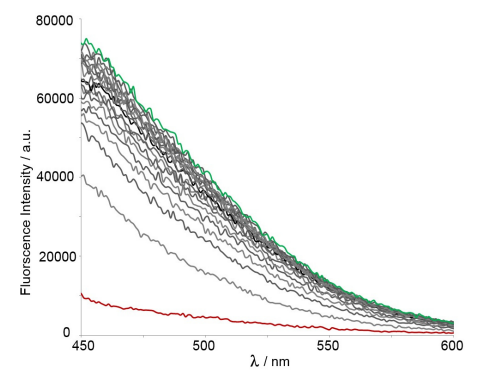


Figure 6. Fluorescence spectrum of $40 \mu \mathrm{M}$ L-DOPA at pH 10 excited at $375 \mathrm{~nm}$ in the presence of (a) No ions and (b) $400 \mu \mathrm{M} \mathrm{CuSO}_{4}$. The first spectrum recorded is marked in red. Subsequent spectra were taken at 3 min intervals with the spectrum at 30 min marked in black and the final spectrum taken at 60 min marked in green.

Recently we have investigated the sheet structure of eumelanin formed from L-DOPA using the sheet-sensing extrinsic probe thioflavin $\mathrm{T}(\mathrm{ThT})$ that has been widely used to investigate sheet structures in beta-amyloid aggregation [7]. The rise of extrinsic fluorescence $I(t)$ due to ThT could be described well by a sigmoidal function with its inherent property to describe the time delay associated with protomolecule formation and subsequent sheet structures [7]. Namely,

$$
I(t)=I(0)+\frac{\alpha}{1+\exp \left(-k\left(t-t_{1 / 2}\right)\right)}
$$

where $I(t)$ is the fluorescence intensity at a time $t, \alpha$ the maximum fluorescence above the background, $t_{1 / 2}$ the time taken for the fluorescence to reached half its maximum and $\mathrm{k}$ the rate of eumelanin formation.

Figure 7(b) shows a typical curve we obtained with ThT fluorescence at $480 \mathrm{~nm}$ in the absence of a metal ion that is described by equation 1 , reflecting a time delay of $t_{1 / 2}=91.6 \mathrm{~min}, k=0.15 \pm 0.05 \mathrm{~min}^{-1}$ at $\mathrm{pH} 10$ as eumelanin sheets are formed [7]. Figure 7(a) compares the growth in intrinsic fluorescence, also at $480 \mathrm{~nm}$, and taken from figure 5(c) without ThT, but with $4 \mathrm{mM}$ of copper present. When fitted to equation 1 the rise in fluorescence caused by the copper yields $k=0.099 \mathrm{~min}^{-1}, t_{1 / 2}=28.2 \mathrm{~min}$ and correlation coefficient $=0.998$. The natural conclusion is that the species giving rise to the $480 \mathrm{~nm}$ fluorescence is formed rapidly and does need either a protomolecule or sheets to be formed and hence represents species formed in the early stage of eumelanin polymerization. Given the known influence of copper in directing dopachrome ring closer towards DHICA rather than DHI [14] it is tempting to attribute the $480 \mathrm{~nm}$ fluorescence to DHICA. However, it is too long a wavelength for DHICA monomer [19, 20].

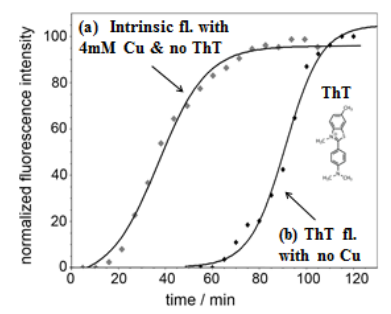

Figure 7. Sigmoidal fit to eumelanin at $480 \mathrm{~nm}$ (a) intrinsic fluorescence with $4 \mathrm{mM} \mathrm{CuSO}_{4}$ added and (b) ThT (inset) fluorescence in the absence of copper. ${ }^{7}$

The strong increase in fluorescence at $480 \mathrm{~nm}$ and decrease at $530 \mathrm{~nm}$ (figure (5)) also correlates with the absorption increase at $450 \mathrm{~nm}$ in the presence of $\mathrm{Cu}^{2+}$ as shown in figure $2 \mathrm{c}$. The addition of $1 \mathrm{mM} \mathrm{Ni}^{2+}$ ions produces a similar effect to $\mathrm{Cu}^{2+}$ giving $k=0.056$ min $^{-1}, t_{1 / 2}=45.21$, min and correlation coefficient $=0.999$.

At $\mathrm{pH} 10$ the hydroxyl, carboxyl and amine groups in eumelanin monomers DHICA and DHI can be deprotonated and hence a colloid stabilized by electrostatic repulsion is produced. At somewhat lower $\mathrm{pH}$ the same concentrations will aggregate more rapidly to produce the brown/black pigment associated with melanin. Hence in addition to the ring closure of dopachrome favouring formation of DHICA rather than DHI [14], the effect of $\mathrm{Cu}^{2+}$ ions is that they can bind to such anionic groups, disturb the stability, and cause the more rapid aggregation as we observe. In the absence of $\mathrm{Cu}^{2+}$ ions at $\mathrm{pH} 9$ dark melanin aggregates are present, but absent at $\mathrm{pH} 10$ as borne out by the transparent (red/brown) solution observed. In the presence of $\mathrm{Cu}^{2+}$ ions at $\mathrm{pH} 10$ the dark aggregated phase returns. 


\subsection{Fluorescence lifetime decay}

Finally, we studied the fluorescence lifetime behaviour in the presence of $\mathrm{Cu}^{2+}$. The fluorescence decay of eumelanin, in various forms, is well-known to be usually complex, often requiring at least three exponentials for a satisfactory description [24-26]. However, we find that in the presence of $\mathrm{Cu}^{2+}$ the fluorescence decay of melanin can be considerably simplified.

(a)

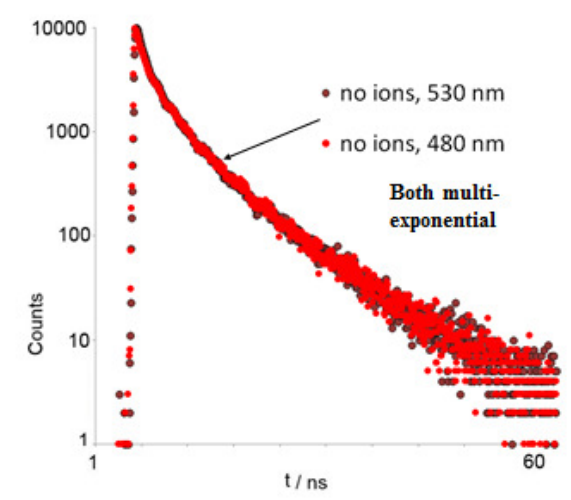

(b)

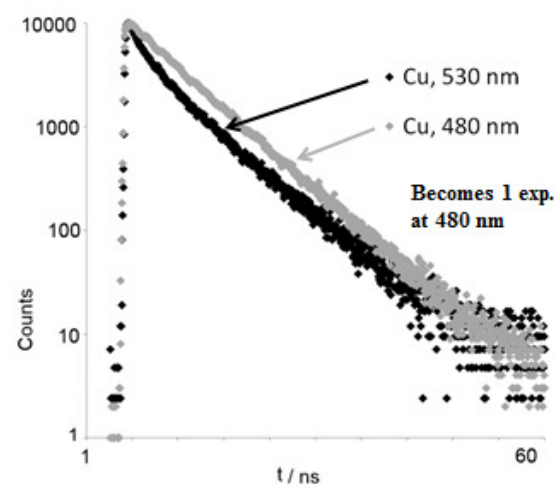

Figure 8. Change of fluorescence decay at $480 \mathrm{~nm}$ and $530 \mathrm{~nm}$ during excitation at $452 \mathrm{~nm}$ (a) without $\mathrm{Cu}^{2+}$ ions and (b) with $4 \mu \mathrm{M} \mathrm{Cu}^{2+}$ ions [23].

The intrinsic fluorescence decay at both $480 \mathrm{~nm}$ and $530 \mathrm{~nm}$ emission requires a three exponential model (equation 2), but in the presence of only $4 \mu \mathrm{M} \mathrm{Cu}^{2+}$ at $530 \mathrm{~nm}$ the fluorescence decay requires a two-exponential function and at 480 $\mathrm{nm}$ a single exponential function (figure 8 , table 1) [23]. A single decay component such as that $\sim 6.33 \mathrm{~ns}$ shown in table 1 suggests only one species is dominant in emitting at this wavelength. When combined with the knowledge that DHICA increases when copper ions is present and yet DHICA monomer cannot be excited at $450 \mathrm{~nm}$ (see figure 2(b)) the logical conclusion is that a single species of oligomer is dominant, and given the early stage nature of the rapid polymerization, possibly a covalently bound dimer or trimer of DHICA/DHI or their oxidised forms. However, at $\mathrm{pH} 10$ DHICA will be deprotonated into a dianion form and the intramolecular excited state proton transfer reaction used to describe eumelanin photophysics at neutral $\mathrm{pH}$ unlikely to be the same [27].

$$
i(t)=B_{1} \exp \left(-t / \tau_{1}\right)+B_{2} \exp \left(-t / \tau_{2}\right)+B_{3} \exp \left(-t / \tau_{3}\right)
$$

Table 1. Fluorescence decay components of eumelanin formed from L-DOPA with and without copper when excited at $452 \mathrm{~nm}$ [23].

\begin{tabular}{|c|c|c|c|c|c|c|}
\hline $\begin{array}{c}\text { Sample, Fl. } \\
\text { Wavelength/nm }\end{array}$ & $\tau_{1} / \mathrm{ns}$ & $B_{1} / \%$ & $\tau_{2} / \mathrm{ns}$ & $B_{2} / \%$ & $\tau_{3} / \mathrm{ns}$ & $B_{3} / \%$ \\
\hline $\mathrm{no} \mathrm{Cu}^{2+}, 480$ & $4.88 \pm 0.12$ & 18 & $1.01 \pm 0.04$ & 23 & $0.03 \pm 0.05$ & 59 \\
\hline $\mathrm{no} \mathrm{Cu}^{2+}, 530$ & $4.87 \pm 0.17$ & 17 & $1.03 \pm 0.17$ & 26 & $0.24 \pm 0.01$ & 57 \\
\hline $4 \mu \mathrm{M} \mathrm{Cu}^{2+}, 480$ & $6.33 \pm 0.03$ & 100 & & & & \\
\hline $4 \mu \mathrm{M} \mathrm{Cu}^{2+}, 530$ & $6.24 \pm 0.03$ & 81 & $1.21 \pm 0.11$ & 19 & & \\
\hline
\end{tabular}


The fluorescence decay of melanin varies strongly with the concentration of $\mathrm{Cu}^{2+}$ present. With the concentration increasing from $4 \mu \mathrm{M}$ to $40 \mu \mathrm{M}$ and $400 \mu \mathrm{M}$ and excitation at $378 \mathrm{~nm}$ the decay is dominated by increasingly longer lifetime components (figure 9(a)) although the overall fluorescence intensity is found to still be reduced. In figure 9(b)

(a)

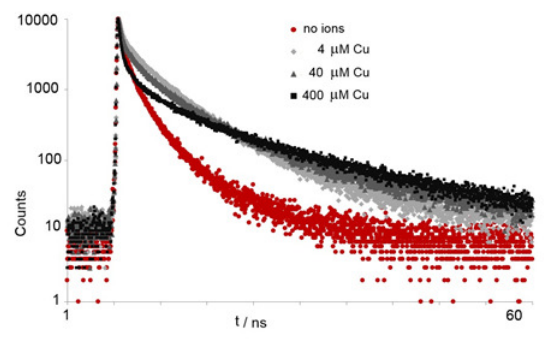

(b)

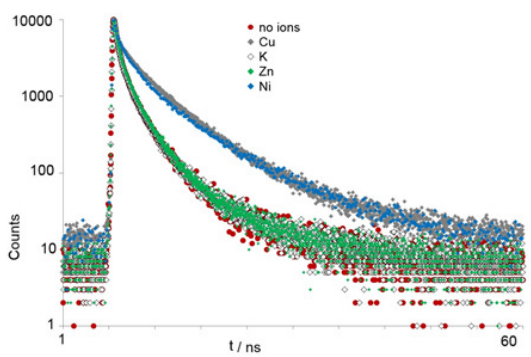

(c)

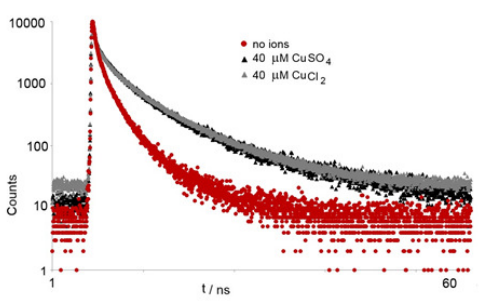

Figure 9. The fluorescence lifetime decay of eumelanin is measured at one hour after the onset of the synthesis at 480 $\mathrm{nm}$ using excitation at $378 \mathrm{~nm}$ with a Delta Diode. (a) With no copper ions present (red dots) and at 4,40 and $400 \mu \mathrm{M}$ of $\mathrm{Cu}^{2+}$. The presence of increasing amounts of $\mathrm{Cu}^{2+}$ during the formation process leads to an increasingly dominant longer decay component. (b) With $4 \mu \mathrm{M} \mathrm{K}^{+}$or $\mathrm{Zn}^{2+}$ ions, which do not alter the fluorescence decay, and with $4 \mu \mathrm{M} \mathrm{Cu}{ }^{2+}$ or $\mathrm{Ni}^{2+}$, each having a similar decay with a longer component of increased intensity. (c) With addition of $40 \mu \mathrm{M} \mathrm{CuCl}_{2}$ compared to $40 \mu \mathrm{M} \mathrm{CuSO}_{4}$ and no metal cation present.

the effect of $\mathrm{Cu}^{2+}$ is compared with $\mathrm{Ni}^{2+}$, which shows a similar decay profile at $4 \mu \mathrm{M}$ concentration, and with $\mathrm{K}^{+}$or $\mathrm{Zn}^{2+}$ which have little if any effect. Similarly figure 9(c) shows that it is the divalent metal ion that is most effective in inducing change as using copper salt with a chloride rather than sulphate anion makes little difference. The complete fluorescence decay analysis in the presence of different copper concentrations and different metal ions is shown in table 2.

Table 2. Fluorescence decay components of eumelanin formed from L-DOPA in the presence of different metal ions at one hour after the onset of the synthesis at $480 \mathrm{~nm}$ using excitation at $378 \mathrm{~nm}$. The chi-squared $\left(X^{2}\right)$ goodness of fit criterion shows that a 3 exponential model provides quite a good description under all the conditions.

\begin{tabular}{|c|c|c|c|c|c|c|c|}
\hline $\begin{array}{c}\text { Sample, Fl. } \\
\text { Wavelength/nm }\end{array}$ & $\tau_{1} / \mathrm{ns}$ & $B_{1}$ & $\tau_{2} / \mathrm{ns}$ & $B_{2}$ & $\tau_{3} / \mathrm{ns}$ & $B_{3}$ & $X^{2}$ \\
\hline no added ions, 480 & $1.87 \pm 0.03$ & 39 & $6.94 \pm 0.15$ & 9 & $0.42 \pm 0.05$ & 30 & 1.210 \\
\hline $4 \mu \mathrm{M} \mathrm{Cu}^{2+}, 480$ & $3.06 \pm 0.06$ & 48 & $7.24 \pm 0.07$ & 28 & $0.48 \pm 0.01$ & 9 & 1.127 \\
\hline $40 \mu \mathrm{M} \mathrm{Cu}^{2+}, 480$ & $3.11 \pm 0.05$ & 42 & $10.16 \pm 0.08$ & 28 & $0.48 \pm 0.01$ & 12 & 1.172 \\
\hline $400 \mu \mathrm{M} \mathrm{Cu}^{2+}, 480$ & $3.16 \pm 0.06$ & 23 & $12.35 \pm 0.08$ & 37 & $0.38 \pm 0.01$ & 14 & 1.010 \\
\hline $4 \mu \mathrm{M} \mathrm{Ni}^{2+}, 480$ & $2.45 \pm 0.05$ & 39 & $7.09 \pm 0.05$ & 35 & $0.48 \pm 0.01$ & 12 & 0.974 \\
\hline $4 \mu \mathrm{M} \mathrm{Zn}^{2+}, 480$ & $1.80 \pm 0.03$ & 41 & $6.22 \pm 0.13$ & 10 & $0.41 \pm 0.01$ & 26 & 1.188 \\
\hline $4 \mu \mathrm{M} \mathrm{K}^{+}, 480$ & $1.83 \pm 0.03$ & 38 & $6.62 \pm 0.13$ & 10 & $0.41 \pm 0.01$ & 29 & 1.100 \\
\hline
\end{tabular}


The key questions highlighted by these results are what is the role of metal ions in the synthesis of eumelanin from LDOPA and what is their effect on the eventual structure? Although it is generally agreed that DHICA, DHI and their redox forms according to $\mathrm{pH}$, are the main constituents, the synthesis is well-known to be complicated and little understood in the presence of metal ions. Clearly the photophysics of the species formed, and certainly their fluorescence, reflect this complexity but, given the importance of copper in melanogenesis, metal ions in pathology and electronic properties metal ions might impart to potential bio-mimicked devices, progress in understanding is sorely needed.

Of course copper is a well-known quencher of fluorescence, but given that the monovalent ions $\mathrm{Na}^{+}$and $\mathrm{K}^{+}$do not increase the fluorescence it might be thought that the growth in fluorescence at $480 \mathrm{~nm}$ as shown in figure 5 can be explained by each divalent $\mathrm{Cu}^{2+}$ ion binding to two deprotonated groups and indeed perhaps playing a role in bridging between sheet structures of eumelanin. However, if the changes in intrinsic fluorescence in the presence of $\mathrm{Cu}^{2+}$ ions we observe correlate with sheet formation from ThT fluorescence, the primary effect of adding copper ions seems to be to reduce the time delay before the sheets start to form, as once started the rates with and without [7] copper are comparable (i.e. $k=0.099$ and $0.15 \pm 0.05 \mathrm{~min}^{-1}$, respectively, c.f. figure 7). Given the oxidative nature of transition metal ions it would not be surprising if the fluorescence results for copper in particular reflect enhanced polymerization of DHICA/DHI, which, circumstantially lead to a single species emitting at $480 \mathrm{~nm}$ as shown by the mono-exponential decay of $\sim 6.3 \mathrm{~ns}$ (Table 1 ) at this wavelength; this species tentatively ascribed here to a small oligomer. Previously a decay constant of $\sim 6$ ns has been observed as one component of a 3 exponential decay description during eumelanin synthesis without copper ions present, at both $\mathrm{pH} 7$ and 10, but no decay component as long as the $\sim 10 \mathrm{~ns}$ and $12 \mathrm{~ns}$ components in the presence of copper ions shown in table 2 was observed even after 14 days after starting the synthesis [26]. The increase in the long decay component (from $~ 7$ to $12 \mathrm{~ns}$ at $4 \mu \mathrm{M}$ to $400 \mu \mathrm{M}$ respectively) with increasing copper concentration shows that copper does not go on facilitating more of this species as a photophysically isolated form or the decay time would stay constant at $\sim 6 \mathrm{~ns}$ with an increasing amplitude $\left(B_{2}\right)$. So for example, our observations would not fit naturally into the interpretation of the model of eumelanin as a polymer of photophysically isolated porphyrins [28] with copper at its centre, but they would be consistent with oligomeric forms of the latter linked by polymerization-induced extended $\pi$-electron conjugation. However, cross-sheet as well as in-plane sheet polymerization cannot be ruled out as the mechanism for increasing $\pi$-electron conjugation and the sources of the fluorescence emission at wavelengths as long as $480 \mathrm{~nm}$ when excited at $450 \mathrm{~nm}$, where simple indole monomers like DHICA cannot be excited. Clearly not all the copper (or other metal ions) in the solution we have studied is interacting with the eumelanin synthesis. Indeed the unusual combination of a reduction in fluorescence and an increase in decay time with increasing copper concentration (figure 4 and table 2) that we observe suggests a dual environment. For example, if excess solvated copper ions in solution quench some of the exposed fluorescent sites while other copper ions enhance the polymerization of fluorescent sites that are shielded from further quenching.

\section{Conclusions}

Quite simply, like many of the questions surrounding melanin in general, the molecular species responsible for the fluorescence emissions observed are, as of now, largely unknown. Undoubtedly fluorescence alone cannot provide all the answers to unravelling the complexity of metal ions and eumelanin. However, we hope that we have further demonstrated here that, rather than often being neglected because of its complexity and low intensity, fluorescence is still a useful analytical tool for studying eumelanin. Moreover, despite the many undoubted complexities and contradictions in interpreting the absorption spectra of eumelanin, fluorescence studies can lead to some simplification that might help point towards a clearer identification of the fundamental building blocks of eumelanin, assist in designing melanin films for bio-inspired devices or indeed in better understanding eumelanin's biological function in the presence of metal ions.

\section{Acknowledgement}

The authors wish to acknowledge the research support of the EPSRC and Scottish Funding Council. 


\section{References}

[1] Faller P 2009 Copper and zinc binding to amyloid- $\beta$ : coordination, dynamics, aggregation, reactivity and metal ion transfer Chem.Bio.Chem. 10 2837-45

[2] Lincoln K M, Richardson T E, Rutter L, Gonzalez P, Simpkins J W and Green K 2012 An N-heterocyclic amine chelate capable of antioxidant capacity and amyloid disaggregation ACS. Chem. Neurosci. 3 919-27

[3] Mason H S 1948 The chemistry of melanin. III. Mechanism of the oxidation of dihydroxy-phenylalanine by tyrosinase J. Bio. Chem. 172 83-99

[4] Cheng J, Moss S C and Eisner M 1994 X-ray characterisation of melanins - II Pigment Cell Res. 7 263-78

[5] Zajac G W, Gallas J M, Cheng J, Eisner M, Moss S C and Alvarado-Swaisgood A E 1994 The fundamental unit of synthetic melanin: verification by tunnelling microscopy of X-ray scattering results Biochim. et Biophys. Acta. 1199 271278

[6] Watt A A R, Bothma J P and Meredith P 2009 The supramolecular structure of melanin Soft Matter $55754-60$

[7] Sutter J, Bidláková, T, Karolin J, and Birch D J S 2012 Eumelanin Kinetics and Sheet Structure App. Phys. Letts. 100 113701 (4 pages)

[8] Meredith P and Sarna T 2006 The physical and chemical properties of eumelanin Pigment. Cell. Res. $19572-594$

[9] Pan T, Li X and Jankovic J 2011 The association between Parkinson's disease and melanoma Int. J. Cancer 128 2251-60

[10] Li J, Zhu M, Manning-Bog A B , Di Monte D A and Fink A L 2004 Dopamine and L-dopa disaggregate amyloid fibrils: implications for Parkinson's and Alzheimer's disease FASEB J. 18 962-4

[11] Belitsky J M, Ellowitz M Z , Lye D and Kilbo A.L 2012 Small molecule modulators of aggregation in synthetic melanin polymerizations Bioorg. Med. Chem. Lett. 22, 5503-07

[12] Ito S 1986 Reexamination of the structure of eumelanin Biochim. et Biophys. Acta. 883 155-161

[13] Hong L, Simon J D 2007 Current understanding of the binding sites, capacity, affinity, and biological significance of metals in melanin J. Phys. Chem. B, 111 7938-7937

[14] Palumbo A, d'Ischia M, Misuraca G, Prota G, and Schultz T M 1988 Structural modifications in biosynthetic melanins induced by metal ions Biochim. et Biophys. Acta 964 193-99

[15] Ambrico M, Ambrico P F, Ligonzo T, Cardone A, Cicco S R, Lavizzera A., Augelli V and Farinola G M 2012 Memory-like behaviour as a feature of electrical signal transmission in melanin-like bio-polymers Appl. Phys. Lett 100 253702

[16] Mostert A B, Powell B J, Gentle I R and Meredith P 2012 On the origin of elctrical conductivity in the bioelectronic material melanin Appl. Phys. Lett 100093701

[17] Birch D J S and Imhof R E 1991 Time-Domain Fluorescence Spectroscopy Using Time-Correlated Single-Photon Counting Topics in Fluorescence Spectroscopy Volume 1 Techniques vol 1, ed J R Lakowicz (Kluwer Academic / Plenum Publishers) pp 1-96

[18] McGuiness CD, Sagoo K, McLoskey D and Birch D J S 2005 Selective excitation of tryptophan fluorescence decay in proteins using a sub-nanosecond $295 \mathrm{~nm}$ light-emitting diode and time-correlated single-photon counting App. Phys. Letts. 86 261911-3

[19] Di J and Bi S 2003 Aluminium ions accelerated the oxidative stress of copper-mediated melanin formation Spectrochim. Acta Part A $\mathbf{5 9} 3075-83$

[20] Jiménez-Cervantes C, Solano F, Kobayashi T, Urabe K, Hearing V J, Lozano J A and García-Borrón J C 1994 A new enzymatic function in the melanogenic pathway J. Biol. Chem. 269 17993-18001

[21] Robinson G M and Smyth M R 1997 Simultaneous determination of products and intermediates of L-DOPA using capilliary electrophoresis with diode-array detection Analyst 122 797-802

[22] Mosca L, De Marco C, Fontana M and Rosei M A 1999 Fluorescence properties of melanins from opioid peptides Biochem. Biophys. Arch. 371 63-9

[23] Birch D J S and Sutter J 2013 The effect of copper on eumelanin photophysics and morphology SPIE Proc. 8587 858705-1 doi: 10.1117/12.999948

[24] Forrest S E, Lamm W C, Millar D P, Nofsinger J B and Simon J D 2000 A model for activated energy transfer within eumelanin aggregates J. Phys. Chem. B. 104 811-4

[25] Birch D J S, Ganesan A and Karolin J 2005 Metabolic sensing using fluorescence Synthetic Metals 155 410-3

[26] McQueenie R, Sutter J, Karolin J, and Birch D J S, 2012 Eumelanin fibrils J. Biomed. Optics. 17075001 
[27] Gauden M et. al. 2008 Role of solvent, pH, and molecular size in excited-state deactivation of key building blocks: implications for melanin pigment stability J. Am. Chem. Soc. 130 17038-43

[28] Kaxiras E, Tsolakidis A, Zonios G and Meng S 2006 Structural model of eumelanin Phys. Rev. Letts. 97218102 